\title{
Prodromalphase der Psoriasis-Arthritis?
}

Eder L et al. Health Care Utilization for Musculoskeletal Issues During the Pre-diagnosis Period in Psoriatic Arthritis - A Population-Based Study. Arthritis Care \& Research 2021; 73 : 680-686. doi:10.1002/acr.24146

Bis zu $30 \%$ der Patienten mit Psoriasis sind von Psoriasis-Arthritis (PsA) betroffen. Eine verzögerte Diagnosestellung ist mit mehr Gelenkschäden und einer schlechteren körperlichen Funktion verbunden. Für PsA gibt es allerdings keinen zuverlässigen Biomarker. Lässt sich dennoch eine Prodromalphase der PsA anhand bestimmter Daten charakterisieren? Eine kanadische Studie prüfte dazu die Inanspruchnahme von Gesundheitsleistungen.

Bisherige Studien konzentrierten sich v. a. auf Selbstberichte. Daten aus der medizinischen Grundversorgung liegen kaum vor. Die Forscherinnern und Forscher in Kanada führten eine bevölkerungsbasierte, gematchte Kohortenstudie unter Verwendung von elektronischen medizinischen Aufzeichnungen und Verwaltungsdaten durch. Ziel war es, Informationen über die Prädiagnosezeit bei PsA zu gewinnen. Dazu wurde die Inanspruchnahme des Gesundheitswesens im Rahmen der Primärversorgung aufgrund muskuloskelettaler Symptome in einem Zeitraum von 5 Jahren vor der Diagnose der PsA erfasst. Die Forscher verglichen diese Daten mit der Inanspruchnahme des Gesundheitswesens von Personen ohne vorherige entzündliche Arthritis. Hausärzte stellten dazu aus ihren Patientendaten eine PsA-Kohorte und eine Vergleichsgruppe ohne vorherige Spondyloarthritis, ankylosierende Spondylitis oder rheumatoide Arthritis zusammen. Die Gruppen wurden hinsichtlich Alter und Geschlecht gematcht. Erfasste Ergebnisse beinhalteten die Inanspruchnahme von Gesundheitsleistungen sowie Kosten im Zusammenhang mit unspezifischen muskuloskelettalen Symptomen während eines 5-Jahres-Zeitraums vor Indexdatum. Insgesamt wurden 462 PsA-Patienten und 2310 gematchte Vergleichspersonen in die Studie einbezogen. Das mittlere Alter betrug 54,2 Jahre, 55,6\% der Patienten waren 
weiblich. Beide Gruppen waren neben Alter und Geschlecht auch gut ausbalanciert hinsichtlich sozioökonomischen Status und Ruralität. Patienten mit PsA wiesen allerdings eine höhere Anzahl an Komorbiditäten auf.

\section{Ergebnisse}

- Die Odds Ratio (OR) in Bezug auf den Besuch eines Hausarztes wegen unspezifischer muskuloskelettaler Symptome bei Patienten mit PsA betrug 2,14 (95\%-KI 1,73-2,64) im Jahr vor dem Indexdatum. Die OR war bis zu 5 Jahre zuvor ähnlich erhöht.

- Die OR der Inanspruchnahme anderer muskuloskelettaler Gesundheitsleistungen (einschließlich Besuchen bei muskuloskelettalen Fachärzten, Gelenkinjektionen, Gelenkbildgebungen und Besuchen in der Notaufnahme) war bei PsA 5 Jahre vor dem Indexdatum erhöht. Die erhöhte Inanspruchnahme von Gesundheitsleistungen wurde demnach bereits 5 Jahre vor Diagnose festgestellt und unterschied sich signifikant von dem Muster, das bei den angepassten Vergleichspersonen beobachtet wurde.

- Die Gesamtkosten und die Kosten für muskuloskelettale Gesundheitsleistungen vor dem Indexdatum waren bei Patienten mit PsA gegenüber den Vergleichspersonen erhöht.

\section{FAZIT}

Laut Autoren sprechen die Ergebnisse dafür, dass eine Prodromalphase der PsA bei einem signifikanten Anteil der Patienten auftritt. Diese sei durch unspezifische muskuloskelettale Symptome gekennzeichnet. Einschränkung sei, dass Patienten ohne entzündliche Arthritis als Vergleich einbezogen wurden, welche nicht der Allgemeinbevölkerung entsprechen würden. Weitere Studien seien erforderlich, um zu überprüfen, ob und wie in dieser Prodromalphase eine frühere PsA-Diagnose gestellt werden könnte. 